\title{
MOUSE HEPATITIS CORONAVIRUS NUCLEOCAPSID PHOSPHORYLATION
}

\author{
Tiana C. White and Brenda G. Hogue*
}

\section{INTRODUCTION}

The nucleocapsid $(\mathrm{N})$ protein of coronaviruses is a major structural component that packages the large viral RNA genome into a helical nucleocapsid within the mature virion. The $\mathrm{N}$ protein is multifunctional. The protein interacts with itself and with the viral $\mathrm{M}$ protein. ${ }^{1,2,3} \mathrm{~N}$ has also been shown to bind viral RNA at both the packaging signal and the 5' leader sequence that is common to both viral genomic RNA and all subgenomic RNAs. ${ }^{4,5,6}$ In addition to its role in virion structure and RNA binding, N has been implicated as playing a role in viral replication. Coronavirus replicons either expressing $\mathrm{N}$ or replicating in the presence of $\mathrm{N}$ protein supplied in trans showed enhanced activity over replicons transfected without the presence of $\mathrm{N}^{7}$ This evidence has led to the general opinion that $\mathrm{N}$ protein is involved in the transcription and/or replication complexes of the virus. Taken together, $\mathrm{N}$ is clearly a dynamic viral protein.

The $\mathrm{N}$ protein is phosphorylated, a feature conserved across the family. Mouse hepatitis virus A59 (MHV), a group II coronavirus, is being used as a model to study $\mathrm{N}$ protein phosphorylation. Data suggest that the $\mathrm{N}$ protein of BCV and MHV exists in at least two phosphorylated forms, indicated by differing molecular weights, during the viral life cycle. ${ }^{8}$ Data also suggests that only one form is packaged into virions. Thus, phosphorylation of the $\mathrm{N}$ protein may play a role in viral assembly. Alternatively, different phosphorylated forms of the protein may perform distinct functions in assembly, replication, and/or transcription. To begin understanding the role that phosphorylation plays in any of the functions provided by $\mathrm{N}$, we have begun identifying which amino acids are phosphorylated in the mature virion and in infected cells. This report focuses on preliminary identification of sites that are phosphorylated in the mature virion. Mass spectrometry is being used to identify which of the many predicted phosphorylation sites within the $\mathrm{N}$ protein are actually modified in the virion and in infected cells. Serine 389 and serine 424 have been preliminarily identified on the $\mathrm{N}$ protein from purified virions

* Arizona State University, Tempe, Arizona. 
as sites that are phosphorylated. Complete mass spectrometry analysis will provide a basis for understanding the role of $\mathrm{N}$ protein phosphorylation.

\section{MATERIALS AND METHODS}

Mouse 17 clone $1(17 \mathrm{Cl1})$ cells were infected with MHV A59 at an MOI of 0.1 . The extracellular media were collected and clarified by centrifugation. The supernatant containing the virions was precipitated with polyethylene glycol (PEG) 8000 in $0.4 \mathrm{M}$ sodium chloride and was purified through a $20-60 \%$ sucrose gradient. Fractions containing virus particles were combined and concentrated by pelleting through a $30 \%$ sucrose cushion. Virions were resuspended in TMEN buffer. Purified virion proteins were analyzed in a $10 \%$ SDS-PAGE gel. The band containing the N protein was removed from the gel, destained, and incubated under reducing conditions with DTT prior to in-gel digestion with proteomics grade porcine pancreatic trypsin (Sigma). Resulting peptides were extracted and dried under vacuum. A portion of the peptide mixture was passed through a gallium (III) immobilized metal affinity column (IMAC) (Pierce) to enrich and isolate phosphopeptides. The IMAC elution was dried under vacuum prior to MALDITOF mass spectrometry analysis.

\section{RESULTS}

Identification of phosphorylation sites began by analyzing tryptic digestion products of N protein from mature virions by MALDI-TOF mass spectrometry. The FindMod tool on ExPASy (http://us.expasy.org/tools/findmod) was used to rapidly identify peaks reported by MALDI that corresponded to predicted peptide masses with an addition of approximately $80 \mathrm{Da}$, representing the addition of one phosphate group. Of the several putative masses identified by the software, only two of the peaks were detectable above the noise level that exhibited normal isotopic patterns (Fig. 1A). The first identified peak had a mass of $555.084 \mathrm{Da}$, corresponding to peptide -NVpSR- encompassing amino acids 422-425. The predicted mass for this peptide is $475.263 \mathrm{Da}$, the experimental mass detected by MALDI results from an additional $79.8217 \mathrm{Da}$, the equivalent of one phosphate group. The second peak that was identified had a mass of $1534.6747 \mathrm{Da}$, corresponded to peptide -DGGADVVpSPKPQRK- which includes amino acids 382-394. This peak was identified by the software as being $80.8023 \mathrm{Da}$ larger than the predicted mass for that peptide.

To confirm that the identified peaks were actually phosphopeptides, total digestion products were bound to an immobilized metal affinity column under highly acidic conditions. Bound peptides were eluted under basic conditions after several wash steps. A mass at 556.242 eluted from the column that exhibited a normal isotopic pattern (Fig. 1B). This peak confirmed that the mass detected earlier at $555 \mathrm{Da}$ was in fact a phosphorylated peptide. 


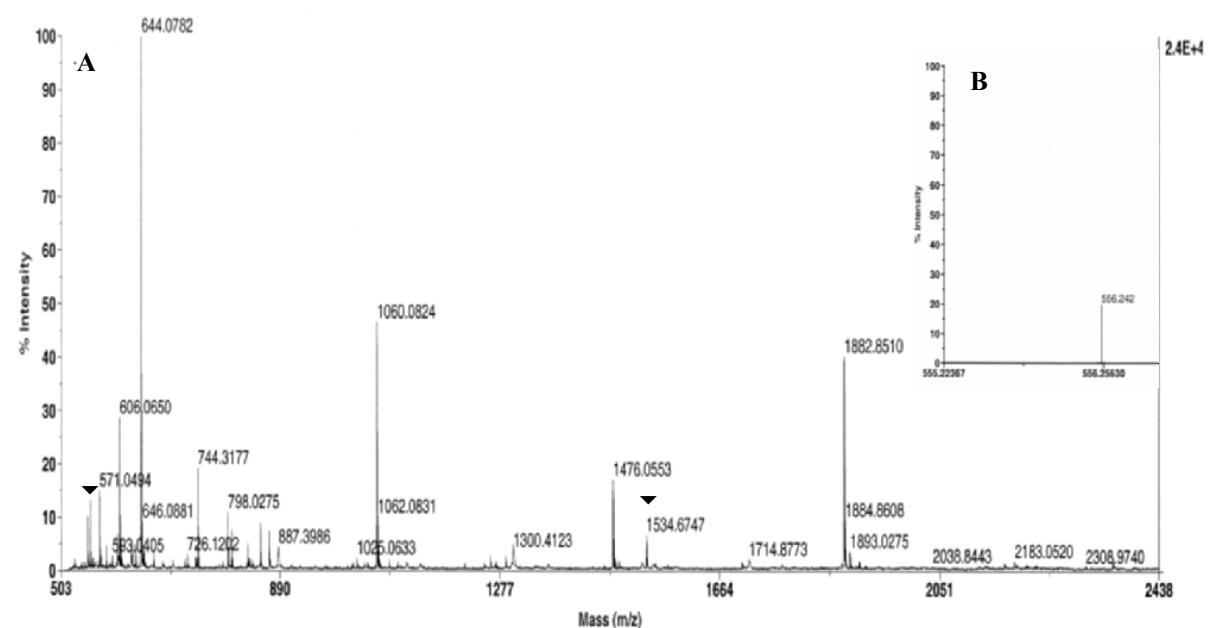

Figure 1. MALDI-TOF spectrum of peptides produced from a tryptic digest of N. The spectra obtained represent an average of 100 laser shots per spectrum. Samples were resuspended in $\alpha$-cyano 4 -hydroxycinnamic acid matrix and shot in positive ion mode with delayed extraction. (A) Arrows indicate masses that were identified as being approximately $80 \mathrm{Da}$ larger than predicted tryptic peptide masses for N. (B) Spectrum obtained after peptides were eluted from gallium (III) IMAC column. A mass at 556.242 Da confirms the mass observed initially as being a phosphopeptide.

\section{DISCUSSION}

Two peaks, with masses 555.084 Da and 1534.578 Da, were identified as possible phosphopeptides from a tryptic digestion of the MHV A59 virion N protein. The peptides were calculated to be approximately $80 \mathrm{Da}$ larger than the predicted peptide masses. The masses of these peaks preliminarily place the phosphorylation sites on the virion $\mathrm{N}$ protein at serine 424 and 389 . However, when peptides were selected by retention on a gallium (III) IMAC column, only one of the sites, serine 424, was confirmed to be phosphorylated. Because the Voyager DE STR MALDI-TOF (Applied Biosystems) mass spectrometer used to obtain the mass information was not equipped with tandem capabilities, the sequence of the peptides could not be confirmed. Therefore, we preliminarily conclude that the serines at positions 424 and 389 are phosphorylated based on the combination of mass spectrometry data, software predictions, and past data illustrating that the $\mathrm{N}$ protein is most likely phosphorylated exclusively on serine residues. ${ }^{9}$ Ongoing tandem mass spectrometry experiments will provide more conclusive identification of these sites.

All coronavirus nucleocapsid proteins are phosphorylated. How conserved the positioning of these sites are remains to be determined. To date, phosphorylation sites for two coronaviruses have been identified. Recombinant $\mathrm{N}$ protein from infectious bronchitis virus, a group III coronavirus, expressed in mammalian cells is phosphorylated at serines 190, 192, 379, and threonine 378. ${ }^{12}$ Transmissible gastroenteritis virus $\mathrm{N}$ protein, a member of group I, isolated from infected cells is phosphorylated at serines 9 , 156,254 , and $256 .^{13}$ The two preliminary sites on the group II MHV presented here are located in the carboxy end of the N protein within domain III. Two of the identified IBV 
sites map at the carboxy end of the protein, whereas none of the identified sites in the TGEV N, from either infected cells or mature virions, are present in the domain III carboxy region. Additionally, both IBV and TGEV have phosphorylated residues in domain II, which also contains the RNA binding domain of $\mathrm{N}^{6,14}$ It remains to be determined if additional sites will be identified for MHV in domain II.

Given that phosphorylation plays an important role in the regulation of both cellular and viral proteins, the role of $\mathrm{N}$ protein phosphorylation remains an intriguing, important area of study. There are precedents for phosphorylation involvement in both virus assembly and RNA transcription. Vesicular stomatitis virus uses phosphorylation as a way to control transcriptional activity. ${ }^{10}$ The import of hepatitis B viral capsids into the nucleus of infected cells is dependent on phosphorylation of the HBV capsid protein. ${ }^{11}$ Thus, identification of phosphorylation sites for both coronavirus intracellular and mature virion $\mathrm{N}$ proteins is well justified.

\section{ACKNOWLEDGMENTS}

This work was supported by Public Health Service grant AI54704, from the National Institute of Allergy and Infectious Diseases.

\section{REFERENCES}

1. L. Kho and P. Masters, Genetic evidence for a structural interaction between the carboxy termini of the membrane and nucleocapsid proteins of mouse hepatitis virus, J. Virol. 76, 4987-4999 (2002).

2. D. Escors, J. Ortego, H. Laude, and L. Enjuanes, The membrane M protein carboxy terminus binds to transmissible gastroenteritis coronavirus core and contributes to core stability, J. Virol. 75, 1312-1324 (2001).

3. K. Narayanan, K. H. Kim, and S. Makino, Characterization of N protein self-association in coronavirus ribonucleoprotein complexes, Virus Res. 98, 131-140 (2003).

4. R. Cologna, J. F. Spagnolo, and B. G. Hogue, Identification of nucleocapsid binding sites within coronavirus-defective genomes, Virology 277, 235-249 (2000).

5. R. Molenkamp and W. Spaan, Identification of a specific interaction between the coronavirus mouse hepatitis virus A59 nucleocapsid protein and packaging signal, Virology 239, 78-86 (1997).

6. G. W. Nelson, S. A. Stohlman, and S. M. Tahara, High affinity interaction between nucleocapsid protein and leader/intergenic sequence of mouse hepatitis virus RNA, J. Gen. Virol. 81, 181-188 (2000).

7. F. Almazán, C. Galán, and L. Enjuanes, The nucleoprotein is required for efficient coronavirus genome replication, J. Virol. 78, 12683-12688 (2004).

8. B. G. Hogue, Bovine coronavirus nucleocapsid protein processing and assembly, Adv. Exp. Med. Biol. 380, 259-263 (1995).

9. S. A. Stohlman and M. Lai, Phosphoproteins of murine hepatitis viruses, J. Virol. 32, 672-675 (1979).

10. S. Barik and A. Banerjee, Sequential phosphorylation of the phosphoprotein of vesicular stomatitis virus by cellular and viral protein kinases is essential for transcription activation, J. Virol. 66, 1109-1118 (1992).

11. B. Rabe, N. Pante, A. Helenius, and M. Kann, Nuclear import of hepatitis B virus capsids and release of the viral genome, Proc. Natl. Acad. Sci. USA 100, 9849-9854 (2003).

12. H. Chen, et. al., Mass spectroscopic characterization of the coronavirus infectious bronchitis virus nucleoprotein and elucidation of the role of phosphorylation in RNA binding by using surface plasmon resonance, J. Virol. 79, 1164-1179 (2005).

13. E. Calvo, D. Escors, J. A. López, J. M. González, A. Álvarez, E. Arza, and L. Enjuanes, Phosphorylation and subcellular localization of transmissible gastroenteritis virus nucleocapsid protein in infected cells, J. Gen. Virol. 86, 2255-2267 (2005).

14. P. Masters, Localization of an RNA-binding domain in the nucleocapsid protein of the coronavirus mouse hepatitis virus, Arch. Virol. 125, 141-160 (1992). 